\title{
Wide-field observations in the SDSS Stripe 82 with the European VLBI Network
}

\author{
H.-M. Cao ${ }^{1,2,5}$, L. I. Gurvits ${ }^{3,4}$, J. Yang ${ }^{3}$, X.-Y. Hong ${ }^{1,5}$, S. Frey ${ }^{6}$, \\ Z. Paragi $^{3}$, A. T. Deller ${ }^{7}$, and Ž. Ivezić $\dot{c}^{8}$ \\ ${ }^{1}$ Shanghai Astronomical Observatory, Chinese Academy of Sciences, 200030 Shanghai, China, \\ email: hmcao@shao.ac.cn \\ ${ }^{2}$ University of Chinese Academy of Sciences, 100049 Beijing, China \\ ${ }^{3}$ Joint Institute for VLBI in Europe, Postbus 2, 7990 AA Dwingeloo, The Netherlands \\ ${ }^{4}$ Department of Astrodynamics and Space Missions, Delft University of Technology, \\ Kluyverweg 1, 2629 HS Delft, The Netherlands \\ ${ }^{5}$ Key Laboratory of Radio Astronomy, Chinese Academy of Sciences, 210008 Nanjing, China \\ ${ }^{6}$ FÖMI Satellite Geodetic Observatory, PO Box 585, H-1592 Budapest, Hungary \\ ${ }^{7}$ ASTRON, The Netherlands Institute for Radio Astronomy, \\ Postbus 2, 7990 AA Dwingeloo, The Netherlands \\ ${ }^{8}$ Department of Astronomy, University of Washington, Seattle, Washington 98195, USA
}

\begin{abstract}
We observed an area of sky located within the SDSS Stripe 82 field at $1.6 \mathrm{GHz}$ with the European VLBI Network (EVN). There are fifteen mJy/sub-mJy radio sources within the primary beam of a typical $30-\mathrm{m}$ class EVN radio telescope. Our aim was to obtain information on compact radio structures of all VLBI-detectable sources within this primary beam area. The source of particular interest is the recently identified radio quasar J222843.54+011032.2 $(\mathrm{J} 2228+0110)$ at $z=5.95$. The data correlation was performed at the EVN software correlator at JIVE (SFXC). Three targets (J2228+0110, J222851.45+011203.4, J222941.76+011428.5) were detected, all three with position offsets not exceeding the $3 \sigma$ accuracy of the original low-resolution radio surveys. The detection rate of $20 \%$ is consistent with other wide-field VLBI experiments carried out recently (e.g. Middelberg et al. 2013). The project presented here demonstrates the ability of EVN in multiple-phase-centre experiments and paves the way for future large-scale EVN surveys of compact structures in extragalactic radio sources using the multiplephase-centre VLBI technique.
\end{abstract}

Keywords. Wide-field VLBI, quasar, survey.

\section{Introduction}

Recently, Zeimann et al. (2011) identified a high-redshift radio quasar J2228+0110. The source is the second radio-selected $z>5.7$ quasar and the fourth $z \sim 6$ quasar with flux density $>1 \mathrm{mJy}$ at $\mathrm{cm}$ radio waves, that makes it a feasible target for VLBI to probe the physical properties at milli-arcsecond (mas) scales of the radio sources located in the early Universe. We observed a field centred midway between J2228+0110 and a strong in-beam phase calibrator J2229+0114 to investigate simultaneously the extremely highredshift object and other fourteen neighbour mJy/sub-mJy radio sources resulted from the overlapping of the Stripe 82 VLA Survey (Hodge et al. 2011), the VLA FIRST Survey (White et al. 1997) and the NVSS (Condon et al. 1998), using the multiple-phase-centre mode (Deller et al. 2011; Morgan et al. 2011) of EVN in a combination with in-beam and nodding phase referencing. 
Table 1. The physical parameters of the three detected sources

\begin{tabular}{lcccccccc}
\hline ID & Name & $\begin{array}{c}S_{V L B I} \\
\mu \mathrm{Jy}\end{array}$ & $\begin{array}{c}\vartheta \\
\text { mas }\end{array}$ & $\begin{array}{c}\theta_{\text {min }} \\
\text { mas }\end{array}$ & $\begin{array}{c}T_{b} \\
10^{8} \mathrm{~K}\end{array}$ & $\begin{array}{c}\text { RA (J2000) } \\
\text { HH:MM:SS }\end{array}$ & $\begin{array}{c}\text { DEC (J2000) } \\
\text { DD:MM:SS }\end{array}$ & $\begin{array}{c}\text { P-Err } \\
\text { mas }\end{array}$ \\
\hline J2228+0110 & $\mathrm{J} 222843.54+011032.2$ & $300 \pm 120$ & $1.71 \pm 0.47$ & 1.30 & $>3.14$ & $22: 28: 43.52679$ & $01: 10: 31.9109$ & 0.59 \\
VLA-4 & $\mathrm{J} 222851.45+011203.4$ & $340 \pm 190$ & - & 1.30 & $>0.89$ & $22: 28: 51.44711$ & $01: 12: 03.4259$ & 0.60 \\
FIRST-3 & $\mathrm{J} 222941.76+011428.5$ & $1000 \pm 460$ & $4.14 \pm 1.72$ & 0.82 & $>0.26$ & $22: 29: 41.76034$ & $01: 14: 27.4236$ & 1.02 \\
\hline
\end{tabular}

Col. 1-Col. 2: source ID used in the project and IAU source name (J2000); Col. 3: flux density recovered by VLBI; Col. 4: the size of the circular Gaussian model at FWHM; a point source model was used to fit the visibilities of VLA-4; Col. 5: minimum resolvable size of each source (Kovalev et al. 2005); Col. 6: brightness temperature; the $T_{b}$ values of VLA-4 and FIRST-3 are estimated with $z=0$ as spectroscopic redshifts are unavailable; the photometric redshift of VLA-4 is $0.74 \pm 0.13$ (SDSS DR9); no obvious optical counterpart of FIRST-3 is found in the SDSS Stripe 82 database. Col. 7-Col. 9: the astrometric position of the source derived by the phase-referencing techniques and the position error

\section{Observations, results and discussion}

The EVN EG057 experiment took place on 2011 Nov 1, with a total observing time of eight hours and a data rate of 1 Gbps per telescope. A ParselTongue script (Kettenis et al. 2006) was used to calibrate the sixteen sets of data simultaneously. A search for EVN detections was conducted in $4^{\prime \prime} \times 4^{\prime \prime}$ areas around low-resolution positions of all targets. The ratios of peak brightness to noise (measured in dirty map with natural weighting) are 12.1, 7.7 and 8.3, for J2228+0110, VLA-4 and FIRST-3 respectively. All of them were observed with VLBI for the first time. The source J2228+0110 was undetected at the $3 \sigma$ image noise level by WSRT observing simultaneously with EVN, which provides a flux-density upper limit of $\sim 0.58 \mathrm{mJy}$. The physical parameters measured by VLBI are listed in Table 1. The three targets appear to be compact, based on the VLBI flux densities obtained in our experiment and the values of VLA peak brightnesses which are $0.31 \pm 0.057,0.37 \pm 0.064,1.53 \pm 0.138(\mathrm{mJy} /$ beam) respectively. The result of the project allows us to conclude that the novel multiple-field-centre technique in combination with in-beam and nodding phase referencing offers an efficient tool for massive VLBI survey of extragalactic radio sources (e.g. Frey et al. 2008; Middelberg et al. 2013).

\section{Acknowledgements}

We acknowledge support from the Royal Dutch Academy of Sciences (KNAW), the Chinese Academy of Sciences (CAS; project n. 10CDP005), the Opening Project of Shanghai Key Laboratory of Space Navigation and Position Techniques (Grant No.13DZ2273300), and the Hungarian Scientific Research Fund (OTKA K104539). The EVN is a joint facility of European, Chinese, South African and other radio astronomy institutes funded by their research councils. Funding for the SDSS and SDSS-II has been provided by the Alfred P. Sloan Foundation, the Participating Institutions, the National Science Foundation, the U.S. Department of Energy, the National Aeronautics and Space Administration, the Japanese Monbukagakusho, the Max Planck Society, and the Higher Education Funding Council for England. The SDSS Web Site is http://www.sdss.org/.

\section{References}

Condon, J. J., Cotton, W. D., Greisen, E. W., et al. 1998, AJ, 115, 1693

Deller, A. T., Brisken, W. F., Phillips, C. J., et al. 2011, PASP, 123, 275

Frey, S., Gurvits, L. I., Paragi, Z., et al. 2008, A\&A, 477, 781

Hodge, J. A., Becker, R. H., White, R. L., Richards, G. T., \& Zeimann, G. R. 2011, AJ, 142, 3

Kettenis, M., van Langevelde, H. J., Reynolds, C., \& Cotton, B. 2006, ASPC, 351, 497

Kovalev, Y. Y., Kellermann, K. I., Lister, M. L., et al. 2005, AJ, 130, 2473

Middelberg, E., Deller, A. T., Norris, R. P., et al. 2013, A\&A, 551, 97

Morgan, J. S., Mantovani, F., Deller, A. T., et al. 2011, A\&A, 526, 140

White, R. L., Becker, R. H., Helfand, D. J., \& Gregg, M. D. 1997, ApJ, 475, 479

Zeimann, G. R., White, R. L., Becker, R. H., et al. 2011, ApJ, 736, 57 